\title{
Highly Scalable Algorithms for Robust String Barcoding ${ }^{\star}$
}

\author{
B. DasGupta ${ }^{1}$, K.M. Konwar² , I.I. Măndoiu² ${ }^{2}$ and A.A. Shvartsman² \\ 1 Department of Computer Science, University of Illinois at Chicago, \\ Chicago, IL 60607-7053 \\ dasgupta@cs.uic.edu \\ 2 Computer Science and Engineering Department, University of Connecticut, \\ 371 Fairfield Rd., Storrs, CT 06269-2155 \\ $\{$ kishori, ion, aas\}@cse.uconn.edu
}

\begin{abstract}
String barcoding is a recently introduced technique for genomicbased identification of microorganisms. In this paper we describe the engineering of highly scalable algorithms for robust string barcoding. Our methods enable distinguisher selection based on whole genomic sequences of hundreds of microorganisms of up to bacterial size on a well-equipped workstation, and can be easily parallelized to further extend the applicability range to thousands of bacterial size genomes. Experimental results on both randomly generated and NCBI genomic data show that whole-genome based selection results in a number of distinguishers nearly matching the information theoretic lower bounds for the problem.
\end{abstract}

\section{Introduction}

String barcoding is a recently introduced technique for genomic-based identification of microorganisms such as viruses or bacteria. The basic barcoding problem [14] is formulated as follows: given the genomic sequences $g_{1}, \ldots g_{n}$ of $n$ microorganisms, find a minimum number of strings $t_{1}, \ldots, t_{k}$ distinguishing these genomic sequences, i.e., having the property that, for every $g_{i} \neq g_{j}$, there exists a string $t_{l}$ which is a substring of $g_{i}$ or $g_{j}$, but not of both. A closely related formulation was independently proposed in [3], where it is assumed that it is possible to detect not just the presence or absence of a distinguisher $t_{i}$, but also the number of repetitions of $t_{i}$ as a substring, up to a threshold of $R>0$. The formulation in [14], which we adopt in this paper, corresponds to $R=1$.

Identification is performed by spotting or synthesizing on a microarray the Watson-Crick complements of the distinguisher strings $t_{1}, \ldots, t_{k}$, and then hybridizing to the array the fluorescently labeled DNA extracted from the unknown microorganism. Under the assumption of perfect hybridization stringency, the hybridization pattern can be viewed as a string of $k$ zeros and ones, referred to as the barcode of the microorganism. By construction, the barcodes corresponding

\footnotetext{
* The work of BD was supported in part by NSF grants CCR-0206795, CCR-0208749 and NSF CAREER grant IIS-0346973. The work of KMK and AAS was supported in part by NSF ITR grant 0121277 . The work of IIM was supported in part by a "Large Grant" from the University of Connecticut's Research Foundation.
} 
to the $n$ microorganisms are distinct, and thus the barcode uniquely identifies any one of them. To improve identification robustness, one may also require redundant distinguishability (i.e., at least $m$ different distinguishers for every pair of microorganisms, where $m>1$ is some fixed constant) and impose a lower bound on the edit distance between any pair of selected distinguishers [14].

The algorithms previously proposed for string barcoding are based on integer programming [14], and on Lagrangian relaxation and simulated annealing [3]. Unfortunately, the run-time of these algorithms does not scale well with the number of microorganisms and the length of the genomic sequences, e.g., the largest instance sizes reported in [14] have a total genomic sequence length of around 100,000 bases.

In this paper we describe the engineering of highly scalable algorithms for robust string barcoding. Our methods enable distinguisher selection based on whole genomic sequences of hundreds of microorganisms of up to bacterial size on a well-equipped workstation, and can be easily parallelized to further extend the applicability range to thousands of bacterial size genomes. Whole-genome based selection is beneficial in at least two significant ways. First, it simplifies assay design since the DNA of the unknown pathogen can be amplified using inexpensive general-purpose whole-genome amplification methods such as specialized forms of degenerate primer multiplex PCR [5] or multiple displacement amplification [9]. Second, whole-genome based selection results in a reduced number of distinguishers, often very close to the information theoretic lower bound of $\left\lceil\log _{2} n\right\rceil$.

Our algorithms are based on a simple greedy selection strategy - in every iteration we pick a substring that distinguishes the largest number of not-yetdistinguished pairs of genomic sequences. This selection strategy is an embodiment of the greedy setcover algorithm (see, e.g., [16]) for a problem instance with $O\left(n^{2}\right)$ elements corresponding to the pairs of sequences. Hence, by a classical result of $[6,11,13]$, our algorithm guarantees an approximation factor of $2 \ln n$ for the barcoding problem. Very recently, Berman et al. [1] have shown that no approximation algorithm can guarantee a factor of $(1-\epsilon) \ln n$ unless $N P=D T I M E\left(n^{\log \log n}\right)$, and also proposed an information content greedy heuristic achieving an approximation factor of $1+\ln n$. Experimental results given in Section 4 show that our setcover greedy algorithm produces solutions of virtually identical quality to those obtained by the information content heuristic.

The setcover greedy algorithm is extremely versatile, and can be easily extended to handle redundancy and minimum edit distance constraints, as well as other biochemical constraints on individual distinguisher sequences. Furthermore, unlike the information content heuristic of [1], the greedy setcover algorithm can also take into account genomic sequence uncertainties expressed in the form of degenerate bases. Although degenerate bases are ubiquitous in genomic databases, previous works have not recognized the need to properly handle them. For example, experiments in [14] have implicitly treated degenerate bases in the input genomic sequences as distinct nucleotides; under this approach a substring of degenerate nucleotides such as NNNNN, might be erroneously selected as a distinguisher although it encodes for any possible substring of length 5 .

To achieve high scalability, our implementation relies on several techniques. First, we use an incremental algorithm for quickly generating a representative set of candidate distinguishers and collecting all their occurrences in the given 
genomic sequences. To reduce the number of candidates, we avoid generating any substring that appears in all genomic sequences, which typically eliminates very short candidates. For each genomic sequence, we also generate only one of the substrings that appear exclusively in that sequence, this optimization eliminates from consideration most candidate distinguishers above a certain length. Unlike the suffix tree method proposed by Rash and Gusfield [14], our approach may generate multiple candidates that appear in the same set of $k$ genomic sequences (for $1<k<n$ ). However, the penalty of having to evaluate redundant candidates in the candidate selection phase is offset in practice by the faster candidate generation time. Finally, the efficient implementation of the greedy selection phase of algorithm combines a partition based method for computing the coverage gain of candidate distinguishers (this method was first proposed in the context of the information content heuristic in [1]) with a "lazy" strategy for updating coverage gains.

The rest of the paper is organized as follows. In Section 2 we give formal problem formulations and review previous work. In Section 3 we describe the efficient implementation of the setcover greedy algorithm for the basic string barcoding problem. In Section 4 we give experimental results on complete microbial genomic sequences extracted from NCBI databases [7].

We conclude in Section 5 with directions for further research.

\section{Preliminaries and Problem Formulation}

Let $\Sigma=\{a, c, g, t\}$ be the DNA alphabet, and $\Sigma^{*}$ be the set of string over $\Sigma$. A degenerate base is a non-empty subset of $\Sigma$. We identify degenerate bases of cardinality 1 with the respective non-degenerate bases. Given a DNA string $x=x_{1} \ldots x_{k} \in \Sigma^{*}$ and a string of degenerate bases $y=y_{1} \ldots y_{n}, n \geq k$, we say that

- $x$ has a perfect match at position $i$ of $y$ iff $y_{i+j-1}=\left\{x_{j}\right\}$ for every $1 \leq j \leq k$,

- $x$ has a perfect mismatch at position $i$ of $y$ iff there exists $1 \leq j \leq k$ such that $\left\{x_{j}\right\} \nsubseteq y_{i+j-1}$, and

- $x$ has an uncertain match at position $i$ of $y$ iff $\left\{x_{j}\right\} \subseteq y_{i+j-1}$ for every $1 \leq j \leq k$, but $y_{i+j-1} \neq\left\{x_{j}\right\}$ for at least one $j$.

String $x=x_{1} \ldots x_{k} \in \Sigma^{*}$ distinguishes two sequences of degenerate bases $y$ and $z$ iff (a) $x$ has a perfect match at one or more positions of $y$, and has perfect mismatches at all positions of $z$, or, symmetrically, (b) $x$ has a perfect match at one or more positions of $z$, and has perfect mismatches at all positions of $y$. The robust string barcoding problem with degenerate bases is formulated as follows: Given sequences of degenerate bases $g_{1}, \ldots g_{n}$ and redundancy threshold $m$, find a minimum number of strings $t_{1}, \ldots, t_{k} \in \Sigma^{*}$ such that, for every $i \neq j$, there exist $m$ distinct strings $t_{l}$ distinguishing $g_{i}$ and $g_{j}$.

It is easy to see that, for $m=1$, at least $\left\lceil\log _{2} n\right\rceil$ distinguishers are needed to distinguish any $n$ genomic sequences. However, achieving this lower bound requires distinguishers that have perfect matches in nearly half of the sequences. In practice, additional constraints, such as lower bounds on the length of distinguishers, may result in no string having perfect matches in a large number of 
sequences, and therefore much more than a logarithmic number of distinguishers. The next theorem establishes, under a simple probabilistic model, that there is an abundance of distinguishers perfectly matching at least a constant fraction of the input sequences; see [8] for the proof.

Theorem 1. Consider a randomly generated instance of the string barcoding problem over a fixed alphabet $\Sigma$ in which there are $n$ strings, each string $s=$ $s_{0} s_{1} \ldots s_{\ell-1}$ is of length exactly $\ell$ generated independently randomly with $\operatorname{Pr}\left[s_{i}=\right.$ $a]=1 /|\Sigma|$ for any $i$ and any $a \in \Sigma$. Also assume that $\ell$ is sufficiently large compared to $n$. Then, for a random string $x \in \Sigma^{*}$ of length $O(\log \ell)$, the expected number of the input strings which contain $x$ as a substring is pn for some constant $0<p<1$.

Previous work. The robust string barcoding problem was introduced (for the case when genomic sequences contain no degenerate bases) by Rash and Gusfield [14]; they provided some experimental results based on integer programming methods, and left open the exact complexity and approximability of this problem. The problem without redundancy constraints was independently considered by Borneman et al. [3], who also considered non-binary distinguishability (based on detecting the multiplicity of a distinguisher as a substring) and a slightly more general problem in which the objective is to pick a given number of distinguishers maximizing the number of distinguished pairs. The main motivation for the formulations in [3] comes from minimizing the number of oligonucleotide probes needed for analyzing populations of ribosomal RNA gene (rDNA) clones by hybridization experiments on DNA microarrays. Borneman et al. provided computational results using Lagrangian relaxation and simulated annealing techniques, and noted that the problem is NP-hard assuming that the lengths of the sequences in the prespecified set were unrestricted. Very recently, Berman, DasGupta and Kao [1] considered a general framework for test set problems that captured the string barcoding problem and its variations; their main contribution is to establish theoretically matching lower and upper bounds on the worst-case approximation ratio. Cazalis et al. [4] have independently investigated similar greedy distinguisher selection strategies for string barcoding. Unlike our work, the algorithms in [4] consider only a small random subset of the possible distinguishers and also prescribe their length in order to achieve practical running time.

\section{Efficient Implementation of the Greedy Setcover Algorithm}

In this section we present the implementation of the setcover greedy algorithm in the context of the basic string barcoding problem, i.e., we disregard redundancy constraints and the presence of degenerate bases in the input sequences. Implementation modifications needed to handle the robust barcoding problem in its full generality are discussed in [8].

Our implementation of the setcover greedy algorithm has two main phases: a candidate generation phase and a candidate selection phase. In the candidate generation phase a representative set of candidate distinguishers is generated from the given genomic sequences. For each generated candidate, we also compute the 


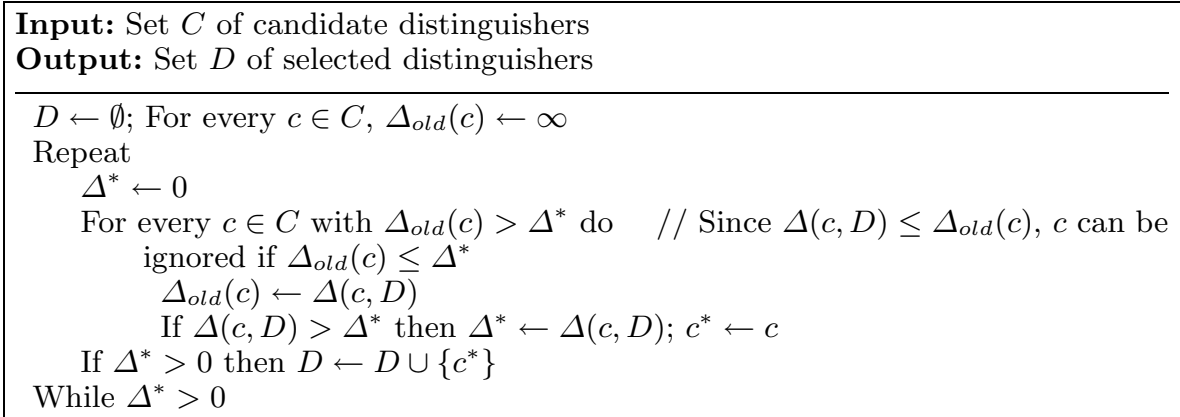

Fig. 1. The greedy candidate selection algorithm

list of sequences with which the candidate has perfect matches; this information is needed in the candidate selection phase. To reduce the number of candidates, we avoid generating any substring that appears in all genomic sequences, which typically eliminates very short candidates. For each genomic sequence, we also make sure to generate only one of the substrings that appear exclusively in that sequence, this optimization eliminates from consideration most candidate distinguishers above a certain length. Unlike the suffix tree method proposed by Rash and Gusfield [14], our approach may generate multiple candidates that appear in the same set of $k$ genomic sequences (for $1<k<n$ ). However, the penalty of having to evaluate redundant candidates in the candidate selection phase is offset in practice by the faster candidate generation time.

Efficient implementation of the above candidate elimination rules is achieved by generating candidates in increasing order of length and using exact match positions for candidates of length $l-1$ when generating candidates of length $l$. For each position $p$ in the input genomic sequences, we also maintain a flag to indicate whether or not the algorithm should evaluate candidate substrings starting at $p$. The possible values for the flag are TRUE (the substring of current length starting at $p$ is a possible candidate), FALSE (we have already saved the substring of current length starting at $p$ as a candidate), or DONE (all candidates containing as prefix the substring of current length starting at $p$ are redundant, i.e., the position can be skipped for all remaining candidate lengths). Initially all flags are set to TRUE. The FALSE flags are reset to TRUE whenever we increment the candidate length, however, we never reset DONE flags. For every candidate length $l$, candidate evaluation proceeds sequentially over all positions of the genomic sequences. Whenever we reach a position $p$ whose flag is set to TRUE, we use the list of matches for the substring of length $l-1$ starting at $p$ (or a linear time string matching algorithm if $l$ is the minimum candidate length) to determine the list of matches for the substring of length $l$ starting at $p$, and set the flag to FALSE for all positions where these matches occur. If the substring of length $l$ starting at $p$ has matches only within the source sequence, and we have already generated a "unique" candidate for this sequence, we discard the candidate and set the flag of $p$ to DONE.

A further speed-up technique is to generate candidate distinguishers from a strict subset of the input sequences. Although this speed-up can potentially affect solution quality, the results in Section 4 show that the solution quality 
loss for whole-genome barcoding is minimal, even when we generate candidates based on a single input sequence, which corresponds to pre-assigning a barcode of all 1's to this sequence.

After the set of candidates is generated we select the final set of distinguishers in the greedy phase of the algorithm (Figure 1). We start with an empty set of distinguishers $D$. While there are pairs of sequences that are not yet distinguished by $D$, we loop over all candidates and compute for each candidate $c$ the number $\Delta(c, D)$ of pairs of sequences that are distinguished by $c$ but not by $D$, then add the candidate $c$ with largest $\Delta$ value to $D$. Two sequences $s$ and $s^{\prime}$ are distinguished by a candidate $c$ iff exactly one of $s$ and $s^{\prime}$ appears in the list $P_{c}$ of perfect matches of $c$, which is available from the candidate generation phase. A simple method for computing $\Delta$ values is to maintain an $n \times n$ symmetric matrix indicating which of the pairs of sequences are already distinguished, and then to probe the $\left|P_{c}\right| \cdot\left(n-\left|P_{c}\right|\right)$ entries in this matrix corresponding to pairs $\left(s, s^{\prime}\right)$ with $s \in P_{c}$ and $s^{\prime} \notin P_{c}$ when computing $\Delta(c, D)$. A more efficient method is based on maintaining the partition defined on the set of sequences by $D$. If the partition defined by $D$ consists of sets $S_{1}, \ldots, S_{k}$, then we can compute $\Delta(c, D)$ in $O\left(k+\left|P_{c}\right|\right)=O(n)$ time using the observation that

$$
\Delta(c, D)=\sum_{i=1}^{k}\left|S_{i} \cap P_{c}\right| \cdot\left|S_{i} \backslash P_{c}\right|
$$

In addition to the fast partition based computation, our implementation of the greedy selection phase uses a lazy strategy for updating the $\Delta$ values, based on the observation that they are monotonically non-increasing during the algorithm (see Figure 1).

\section{Experimental Results}

We performed experiments on both randomly generated instances and whole microbial genomes extracted from the NCBI databases [7]. Due to space constraints we report here only results obtained by the setcover greedy algorithm on microbial genomes. Experimental results comparing the setcover greedy algorithm, the information content heuristic in [1], and a recently proposed multi-step rounding algorithm for set multicover [2] on large number of random testcases are given in $[8]$.

The results on the NCBI dataset, which consists of a set of 29 complete microbial genomic sequences extracted from [7], are given in Table 1. Sequence lengths in the set vary between 490 Kbases and 4.75 Mbases, with an average length of 2.6 Mbases (over 76 Mbases total). The sequences contain a small number of degenerate bases, 861 bases in total. Therefore, we cannot use the partition method for computing the number of sequence pairs distinguished by a candidate in the greedy selection phase, and we have to use the slower matrix datastructure. In these experiments we varied the redundancy requirement from 1 to 20 . To see the effect of length and edit distance requirements on the number of distinguishers, for each redundancy requirement we computed both an unconstrained solution, and a solution in which distinguishers must have length between 15 and 40, and there should be a minimum edit distance of 6 between 
Table 1. Results on a set of 29 NCBI complete microbial genomes. Candidate generation time is approximately 335 seconds for all combinations of parameters. The experiments were run on a PowerEdge 2600 Linux server with 4 Gb of RAM and dual $2.8 \mathrm{GHz}$ Intel Xeon CPUs - only one of which is used by our sequential algorithms

\begin{tabular}{|c|rcccc|}
\hline Redundancy & $l_{\min }$ & $l_{\max }$ & MinEdit & Select time & \#Distinguishers \\
\hline 1 & 0 & $\infty$ & 0 & 14.2 & 6.0 \\
1 & 15 & 40 & 6 & 2.6 & 8.0 \\
\hline 5 & 0 & $\infty$ & 0 & 20.3 & 21.0 \\
5 & 15 & 40 & 6 & 8.7 & 31.0 \\
\hline 10 & 0 & $\infty$ & 0 & 22.9 & 41.0 \\
10 & 15 & 40 & 6 & 16.4 & 60.0 \\
\hline 20 & 0 & $\infty$ & 0 & 26.8 & 76.0 \\
20 & 15 & 40 & 6 & 33.4 & 123.0 \\
\hline
\end{tabular}

every two selected distinguishers (these values are similar to those used in [14]. In all experiments, we generated candidates based only on the shortest sequence of 490 Kbases.

Results in Table 1 show that, naturally, meeting higher redundancy constraints requires more distinguishers to be selected. Additional length and edit distance constraints further increase the number of distinguishers, but the latter is still within reasonable limits. The length constraints reduce the number of candidates (from $1,775,471$ to 122,478 ), which, for low redundancy values has the effect of reducing greedy selection time. However, for high redundancy requirements the reduction in number of candidates is offset by the increase in solution size, and greedy selection becomes more time consuming with length and edit distance than without (selection time grows roughly linearly with solution size).

\section{Conclusions}

In this paper we have given highly scalable algorithms for the robust string barcoding problem, and have shown that distinguisher selection based whole genomic sequences results in a number of distinguishers nearly matching the information theoretic lower bounds for the problem.

In ongoing work we are exploring heuristics and approximation algorithms for several extensions of the string barcoding problem. First, we are considering the use of probe mixtures as distinguishers. With most microarray technologies it is feasible to spot/synthesize a mixture of oligonucleotides at any given microarray location. The DNA of a pathogen will hybridize to such a location if it contains at least one substring which is the Watson-Crick complement of one of the oligonucleotides in the mixture. Using oligonucleotide mixtures as distinguishers can reduce the number of spots on the array - and therefore barcode length - closer to the information theoretical lower-bound of $\log _{2} n$. The reduction promises to be particularly significant when reliable hybridization requires relatively long distinguishers; in these cases even the optimum barcoding length is far from $\log _{2} n$ [14]. A special case of this approach is the use of degenerate distinguishers similar to the degenerate primers that have been recently employed 
in multiplex PCR amplification $[12,15]$. Degenerate distinguishers are particularly attractive for string barcoding since their synthesis cost is nearly identical to the synthesis cost of a single non-degenerate distinguisher (synthesis requires the same number of steps, the only difference is that multiple nucleotides must be added in some of the synthesis steps).

In many practical pathogen identification applications collected biological samples may contain the DNA of multiple pathogens. This issue is considered to be particularly significant in medical diagnosis applications, see, e.g., [10] for studies in detecting more than one HPV (human papiloma virus) genotype with varying rate of multiple $\mathrm{HPV}$ infections carried by the same HPV carrier. In future work we plan to develop extensions of the barcoding technique that can reliably detect multiple pathogens for a given bound on the number of pathogens present.

\section{References}

1. P. Berman, B. DasGupta, and M.-Y. Kao. Tight approximability results for test set problems in bioinformatics. In Proc. 9th Scandinavian Workshop on Algorithm Theory (SWAT), volume 3111 of Lecture Notes in Computer Science, pages 39-50, Berlin, 2004. Springer-Verlag.

2. P. Berman, B. DasGupta, and E. Sontag. Randomized approximation algorithms for set multicover problems with applications to reverse engineering of protein and gene networks. In Proc. 7th International Workshop on Approximation Algorithms for Combinatorial Optimization Problems (APPROX), volume 2748 of Lecture Notes in Computer Science, pages 39-50, Berlin, 2004. Springer-Verlag (To appear in Discrete Applied Mathematics.).

3. J. Borneman, M. Chrobak, G.D. Vedova, A. Figueora, and T. Jiang. Probe selection algorithms with applications in the analysis of microbial communities. Bioinformatics, 1:1-9, 2001.

4. D. Cazalis, T.Milledge, and G. Narasimhan. Probe selection problem: Structure and algorithms. In Proc. 8th Multi-Conference on Systemics, Cybernetics and Informatics (SCI 2004), pages 124-129, 2004.

5. V.G. Cheung and S.F. Nelson. Whole genome amplification using a degenerate oligonucleotide primer allows hundreds of genotypes to be performed on less than one nanogram of genomic dna. Proc. Natl. Acad. Sci. USA., 93:14676-14679, 1996.

6. V. Chvátal. A greedy heuristic for the set covering problem. Math. of Op. Res., 4:233-235, 1979.

7. NCBI Completed Microbial Genomes. http://www.ncbi.nlm.nih.gov/genomes/microbes/complete.html, 2004.

8. B. DasGupta, K. Konwar, I.I. Mandoiu, and A. Shvartsman. Highly scalable algorithms for robust string barcoding. ACM Computing Research Repository, cs.DS/0502065, 2005.

9. F.B. Dean, S. Hosono, L. Fang, X. Wu, A. Fawad Faruqi, P. Bray-Ward, Z. Sun, Q. Zong, Y. Du, J. Du, M. Driscoll, W. Song, S.F. Kingsmore, M. Egholm, and R.S. Lasken. Comprehensive human genome amplification using multiple displacement amplification. Proc. Natl. Acad. Sci. USA., 99:5261-5266, 2002.

10. B. Gharizadeh, M. Käller, P. Nyrén, A. Andersson, M. Uhlén, J. Lundeberg, and A. Ahmadian. Viral and microbial genotyping by a combination of multiplex competitive hybridization and specific extension followed by hybridization to generic tag arrays. Nucleic Acids Research, 31(22), 2003.

11. D.S. Johnson. Approximation algorithms for combinatorial problems. J. Comput. Sys. Sci., 9:256-278, 1974. 
12. C. Linhart and R. Shamir. The degenerate primer design problem. Bioinformatics, 18:S172-S181, 2002.

13. L. Lovász. On the ratio of optimal integral and fractional covers. Discrete Mathematics, 13:383-390, 1975.

14. S. Rash and D. Gusfield. String barcoding: Uncovering optimal virus signatures. In Proc. 6th Annual International Conference on Computational Biology, pages 254-261, 2002.

15. R. Souvenir, J. Buhler, G. Stormo, and W. Zhang. Selecting degenerate multiplex PCR primers. In Proc. 3rd Intl. Workshop on Algorithms in Bioinformatics (WABI), pages 512-526, 2003.

16. V.V. Vazirani. Approximation Algorithms. Springer-Verlag, Berlin, 2001. 